\title{
REDESCRIPCIÓN Y DESIGNACIÓN DE UN NEOTIPO DE PANOCHTHUS TUBERCULATUS (OWEN) (MAMMALIA, CINGULATA, GLYPTODONTIDAE)
}

\author{
MARTÍN ZAMORANO \\ Departamento Científico de Paleontología de Vertebrados, Facultad de Ciencias Naturales y Museo, Universidad Nacional \\ de La Plata, CONICET, Paseo del Bosque s/n, 1900, La Plata. Argentina. marzamorano@fcnym.unlp.edu.ar \\ ÁLVARO MONES \\ Franzensbadstraße 7b, 86199, Augsburg, Alemania.amones@adinet.com.uy
}

GUSTAVO J. SCILLATO-YANÉ

Departamento Científico de Paleontología de Vertebrados, Facultad de Ciencias Naturales y Museo, Universidad Nacional de La Plata, CONICET, Paseo del Bosque s/n, 1900, La Plata, Argentina. scillato@fcnym.unlp.edu.ar

\begin{abstract}
REDESCRIPTION AND DESIGNATION OF A NEOTYPE OF PANOCHTHUS TUBERCULATUS (OWEN) (MAMMALIA, CINGULATA, GLYPTODONTIDAE). We design a neotype for Panochthus tuberculatus (Owen), given that the holotype is missing. A diagnosis and detailed description of the neotype is provided. Glyptodon tuberculatus is the type species of Panochthus Burmeister, one of the genera of giant Pleistocene glyptodonts. P. tuberculatus is recorded in the Bonaerean (late middle Pleistocene) and Lujanian (thus far only for the late Pleistocene lapse of this age) of Argentina, Uruguay, Brazil, Paraguay and, with doubts, Bolivia. Firstly, the species was recognized on the basis of two fragments of dorsal armor; this type material (two syntypes) must be considered as lost, given that it has not been found in the collections of British Museum Natural History, where it was deposited. It is essential that material assigned to P. tuberculatus be made available, to establish the validity of each of the 19 species referred to this genus. The selected material is the complete specimen MLP 16-29, from the Bonaerean of Tapalqué (Buenos Aires Province, Argentina). The specimen of P. tuberculatus MLP 16-29 fulfills all the conditions required by the International Code of Zoological Nomenclature and the designed neotype satisfies all the required subsections. The conclusions are: (i) the specimen MLP 16-29, consisting of nearly complete skeleton and armor of a glyptodontid, until now referred to P. morenoi, becomes the neotype of $P$. tuberculatus; and (ii) consequently, P. morenoi (sensu Ameghino, 1889), is a synonym of P. tuberculatus.
\end{abstract}

Key words: Panochthus tuberculatus, neotype, Pleistocene, Argentina, Glyptodontidae.

RESUMO - O holótipo de Panochthus tuberculatus (Owen) está extraviado. Portanto, um neótipo para esta espécie é aqui designado, diagnosticado e descrito em detalhe. Glyptodon tuberculatus é a espécie tipo de Panochthus Burmeister, um dos gêneros de gliptodontes gigantes do Pleistoceno. P. tuberculatus está presente no Bonaerense (Pleistoceno médio tardio) e Lujanense (até o momento somente para o lapso do Pleistoceno tardio desta idade) da Argentina, Uruguai, Brasil, Paraguai e, com dúvidas, Bolívia. A espécie foi reconhecida primeiramente com base em dois fragmentos dorsais de carapaça; este material tipo (dois síntipos) deve ser considerado perdido, já que não tem sido possível encontrá-lo nas coleções do British Museum Natural History, onde foi depositado. É imprescindível contar com material assignável a P. tuberculatus para determinar quais das 19 espécies mencionadas dentro do gênero são válidas. O material designado é o exemplar completo MLP 16-29, do Bonaerense de Tapalqué (Província de Buenos Aires, Argentina), pois o mesmo cumpre com todas as condiciones exigidas pelo Código Internacional de Nomenclatura Zoológica vigente e todos os incisos requeridos. Conclui-se que: (i) o exemplar MLP 16-29, constituído de esqueleto e carapaça quase completos de um gliptodontídeo até agora atribuído a P. morenoi, passa a ser o neótipo de P. tuberculatus; e (ii) consequentemente, P. morenoi (sensu Ameghino, 1889) resulta ser um sinônimo de P. tuberculatus.

Palavras chave: Panochthus tuberculatus, neótipo, Pleistoceno, Argentina, Glyptodontidae.

\section{INTRODUCCIÓN}

Panochthus Burmeister, 1866, es uno de los géneros de gliptodontes pleistocenos de tamaño gigantesco (Fariña, 2000). Está presente desde el Ensenadense (Pleistoceno temprano tardío) hasta el Lujanense (hasta ahora sólo en el lapso Pleistoceno tardío de esta Edad) en Argentina, Uruguay, Brasil, Bolivia y Perú (Scillato-Yané et al., 1995).
Panochthus tuberculatus (Owen, 1845), la especie tipo, se encuentra en el Bonaerense (Pleistoceno medio tardío) y Lujanense (Pleistoceno tardío, vide supra) de Argentina, Uruguay, Brasil, Paraguay y, con dudas, Bolivia.

La especie tipo del género Panochthus es Glyptodon tuberculatus Owen, 1845. Burmeister reconoce diferencias suficientes como para fundar un nuevo género, aunque en su descripción original (Burmeister, 1866:190-191; 1867:529) 
lo propone como un subgénero de Glyptodon. Recién años más tarde, el mismo Burmeister (1872) eleva Panochthus al nivel de género (Mones, 1994).

Owen (1845:120, lám. 5, figs. 3-5) reconoce la especie Glyptodon tuberculatus basándose en dos trozos de coraza dorsal, "From the tertiary deposits in the Pampas of Buenos Ayres". El material tipo (dos sintipos) debe considerarse perdido, ya que, pese a los esfuerzos realizados, no ha sido posible hallarlo en las colecciones del British Museum Natural History (BMNH) (Mones, 1986:238; Current, com. pers. a M.Z., 2010), donde oportunamente fue parcialmente depositada la colección paleontológica del Royal College of Surgeons de Londres. Lydekker (1887:116) menciona un calco, BM(NH) M-3609, que podría corresponder a uno de los sintipos (¿RCS 3609 ?), pero que tampoco pudo ser hallado. Tras la meticulosa e infructuosa búsqueda del material tipo y con la imperiosa necesidad de contar con material indudablemente asignable a $P$. tuberculatus, para poder determinar así cuáles de las 19 especies mencionadas dentro del género (Mones, 1986:237-238) son válidas y cuáles no (Tabla 1), resulta imprescindible designar un neotipo, objetivo de este trabajo. La diagnosis de esta especie (hasta ahora inexistente), aquí elaborada, la detallada descripción actualizada del material propuesto como neotipo y la comparación de estructuras anatómicas homólogas, tanto con especies congéneres como también con las de otros géneros de gliptodóntidos es absolutamente necesaria para cumplir con el objetivo señalado. El propósito de esta designación se ajusta perfectamente a los requerimientos del Código Internacional de Nomenclatura Zoológica (ICNZ, 1999, Art. 75) y no se corresponde a las "Circunstancias excluidas" (ICNZ, 1999, Art. 75.2).

\section{MATERIAL Y MÉTODOS}

En el primer material en el cual se pensó, y que hubiese sido el candidato ideal para designar como neotipo, fue el ejemplar casi completo que describió y figuró detalladamente Burmeister (1874:2-156, láms. I-XVI). Lamentablemente en ese trabajo, y en ningún otro, se indican los datos del repositorio ni del número de este material. Cuando Burmeister realizó esta descripción se encontraba trabajando en el antiguo Museo Público de Buenos Aires (hoy Museo Argentino de Ciencias Naturales "Bernardino Rivadavia”, MACN). Sin embargo, las colecciones del MACN fueron revisadas minuciosamente y no hay ningún ejemplar identificable como el que utilizó Burmeister (1874).

En el Museo de Ciencias Naturales de La Plata (MLP) se encuentra en exhibición el ejemplar completo MLP 16-29, procedente del Bonaerense de Tapalqué, centro de la Provincia de Buenos Aires (Figura 1). Ameghino (1895:177; 1920:576-577)

Tabla 1. Lista de las especies mencionadas de Panochthus por Mones (1986:237-238), con actualizaciones publicadas en contribuciones previas a ésta.

Table 1. List of the species mentioned of Panochthus by Mones (1986: 237-238), with updates published in previous contributions to this one.

Especies de Panochthus

P. tuberculatus (Owen, 1845)

P. bullifer Burmeister, 1874 (actualmente Propanochthus [ver Castellanos, 1925])

P. lundii Burmeister, 1874

P. morenoi Ameghino, 1881b

P. eocenus Scalabrini, 1887 nomen nudum

P. beyrichi Roth, 1888 nomen nudum

P. brocherii Moreno, 1888 nomen nudum

P. trouessarti Moreno, 1888 (actualmente Phlyctaenopyga [ver Zamorano et al., 2011])

P. vogti Roth, 1888 nomen nudum

P. (?) nodotianus Ameghino, 1889 (Ameghino [1889:837] coloca (?) antes del epíteto especifico, en cambio Mones [1886:238] pone ? antes del género)

P. frenzelianus Ameghino, 1889

P. voghti Ameghino, 1889

P. intermedius Lydekker, 1895

P. subintermedius Castellanos, 1933 nomen nudum (válida desde [Castellanos, 1937] [ver Cruz et al., 2011; en esta publicación hay un error bibliográfico, 1936 en vez de 1937])

P. greslebini Castellanos, 1942

P. oliveiraroxoi Castellanos, 1942 (actualmente sinónimo de P. greslebini [ver Moreira (1971:551) y Porpino \& Bergqvist (2002:52)])

P. rusconii Castellanos, 1942

P. rochai Paula Couto, 1954 (actualmente sinónimo de P. greslebini [ver Moreira (1971:551) y Porpino \& Bergqvist (2002:52)])

P. jaguaribensis Moreira, 1965 
asignó este material a la especie Panochthus morenoi Ameghino, 1881 (originalmente con la grafía "morenii"; el autor de la enmienda, válida, aunque no explicitada, es Lydekker, 1887:125). Si bien a esta última especie se la viene considerando sinónimo de P. tuberculatus (véase Zamorano, 2009; la mencionada identidad será examinada en la Tesis Doctoral, en preparación, de este último autor), para los fines de esta comunicación alcanza con confirmar que en el material MLP 16-29 no se observan las características señaladas por Ameghino (1881a,b) para el material tipo de $P$. morenoi, también perdido, cuya localidad tipo es Uruguay, Bahía de Montevideo: "Las placas de la coraza de esta especie se distinguen de las otras por sus arealitas rudimentarias unidas unas á otras por asperosidades. Los surcos que dividen las arealitas apenas están marcados, y toda la superficie de las placas presenta un gran número de agujeros aunque de pequeño diámetro" (Ameghino, 1881a:309; 1881b:309). Fue Lydekker (1895:36-39, láms. XX-XXIII) el primero que asignó el ejemplar MLP 16-29 a P. tuberculatus; con lo cual coincide y sirve de precedente a la decisión adoptada en esta comunicación.

El material (MLP 16-29) que se designa como neotipo de Panochthus tuberculatus, consiste en un ejemplar muy completo y muy bien conservado. Si bien este material fue descripto

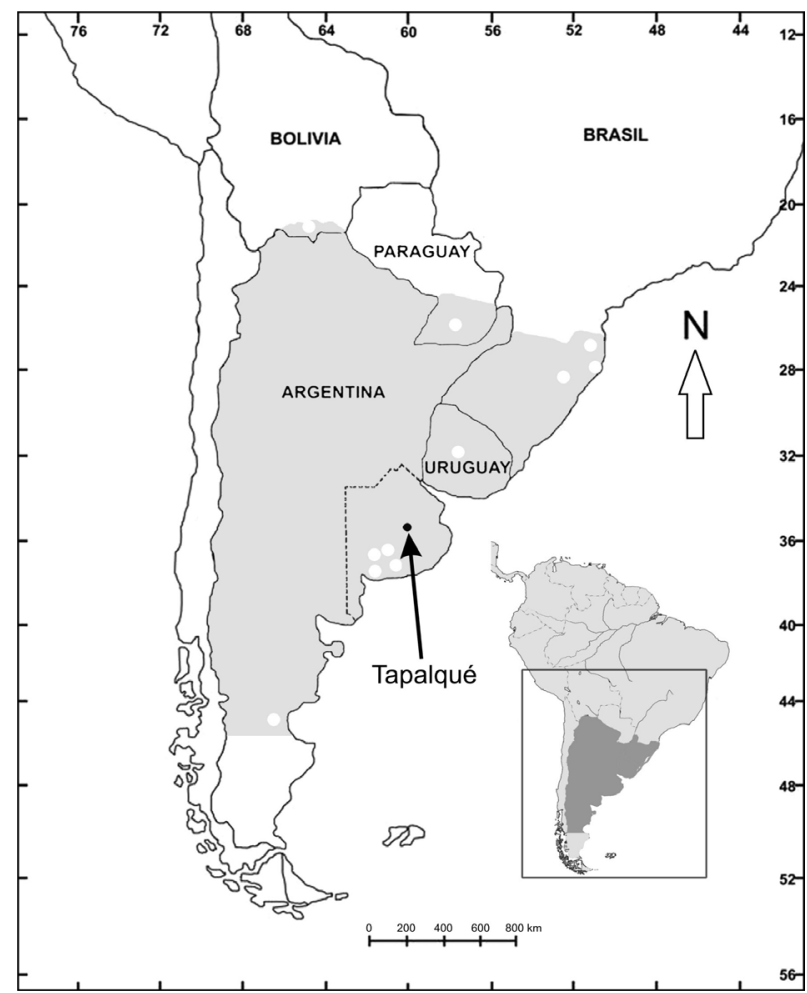

Figura 1. Distribución geográfica de Panochthus tuberculatus durante el Bonaerense y Lujanense (Pleistoceno medio y tardío). El neotipo procede del Bonaerense (Pleistoceno medio) de Tapalqué, Buenos Aires, Argentina (flecha). Círculos blancos, localidades con información precisa de registro; sombreado gris, distribución generalizada sobre la base de datos inéditos y de catálogos.

Figure 1. Geographical distribution of Panochthus tuberculatus during Bonaerian and Lujanian age (middle and late Pleistocene). The neotype comes from the Bonaerian (middle Pleistocene) of Tapalqué, Buenos Aires, Argentina (arrow). White circles, localities with precise information of registry; shaded gray, distribution generalized on the published data and catalogues. detalladamente por Castellanos (1942:531-541, figs. 215-218), en esta comunicación se brinda una redescripción empleando una terminología más actualizada y apropiada. Para describir el material y compararlo con el de especies afines se empleó la terminología anatómica utilizada por Sisson \& Grossman (1959). Asimismo, para características peculiares de los gliptodontes, se consultó a Burmeister (1874), Gillete \& Ray (1981) y Zurita (2007).

Para el tratamiento bioestratigráfico y cronológico se sigue a Cione \& Tonni (2005).

Abreviaturas institucionales. BM(NH), British Natural History Museum, Londres, Inglaterra; MACN, Museo Argentino de Ciencias Naturales "Bernardino Rivadavia", Buenos Aires, Argentina; MLP, Facultad de Ciencias Naturales y Museo de La Plata, La Plata, Argentina; RCS, Royal College of Surgeons Museum, Londres, Inglaterra.

\section{SISTEMÁTICA PALEONTOLÓGICA}

Superorden XENARTHRA Cope, 1889

Orden CINGULATA Illiger,1811

Superfamilia GLYPTODONTOIDEA Gray, 1869

Familia GLYPTODONTIDAE Gray, 1869

Subfamilia HOPLOPHORINAE Huxley, 1864

Tribu PANOCHTHINI Castellanos, 1927

Panochthus Burmeister, 1866

Especie tipo. Glyptodon tuberculatus Owen, 1845.

Sinonimia. Glyptodon Owen, 1838, non Owen, 1839; Schistopleurum Nodot, 1855; Glyptodon (Panochthus) Burmeister, 1866; Panochthus Burmeister, 1872, nuevo rango; Parapanochthus Moreira, 1971 (especie tipo: Panochthus jaguaribensis Moreira, 1965. Sinónimo de Panochthus según Bergqvist [1993]; ver Porpino \& Bergqvist [2002]).

\section{Panochthus tuberculatus (Owen, 1845) Burmeister, 1866} (Figuras 2-4)

1855 Schistopleurum tuberculatum (Owen). Nodot, p.335-366. 1857 Glyptodon verrucosus Nodot, p.100-101, lám. 12, figs. 6-7. 1866 Glyptodon (Panochthus) tuberculatus (Owen). Burmeister, p. 190, 192-194.

1889 Panochthus morenii Ameghino, p. 834, partim emend. illegitima, lám. 65, figs. 1-3 non 1881a P. morenoi Ameghino, p. 309. La eventual sinonimia de P. morenii Ameghino, 1881b, $P$. voghti Ameghino, 1889, y P. rusconii, Castellanos, 1942, con P. tuberculatus, es discutida en la Tesis Doctoral (inédita) de uno de nosotros (M. Z.).

Holotipo. RCS 3607 (ex RCS 558); RCS 3609 (ex RCS 559); corresponderían a dos trozos de coraza dorsal [Extraviados]. Procedencia estratigráfica y geográfica del holotipo. Procedente de depósitos "terciarios" en las Pampas de Buenos Aires ["From the tertiary (sic) deposits in the Pampas of Buenos Ayres"], son los únicos datos que brinda Owen (1845: 120).

Neotipo. MLP 16-29: cráneo, mandíbula, esqueleto postcraneal muy completo, coraza dorsal, anillos caudales y tubo caudal. Localidad tipo determinada por el neotipo. Bonaerense (Pleistoceno medio) de Tapalqué (Partido de Tapalqué, centro 
de la Provincia de Buenos Aires, 36 $20^{\circ} \mathrm{S}$ y $60^{\circ} 01^{\prime} \mathrm{O}$ ) (Figura 1) (ver Ameghino, 1895, 1898; Figini et al., 1998).

Distribución estratigráfica y geográfica: Bonaerense (Pleistoceno medio tardío) y Lujanense (Pleistoceno tardío, vide supra) de Argentina (regiones chacopampeana, mesopotámica, subandina y patagónica septentrional, el límite austral es la localidad de Fitz Roy, Departamento Deseado, Provincia de Santa Cruz [Tauber \& Palacios, 2007]); Uruguay (Mones \& Francis, 1973:53-54), sur de Brasil (Rio Grande do Sul [Kerber \& Oliveira, 2008]), Paraguay (Hoffstetter, 1978) y Bolivia (valle de Tarija, como P. cf. tuberculatus [Hoffstetter, 1963, 1964; Zurita et al., 2009] o como Panochthus sp. [Tonni et al., 2009]) (Figura 1).

Diagnosis. Cráneo muy abovedado, nasales oblicuamente descendentes hacia delante; grado de neumatización mayor que en Panochthus frenzelianus Ameghino, 1889, y mucho mayor que en Panochthus sp. del Ensenadense de Argentina. Fosas orbitaria y temporal separadas por un proceso postorbital completo en vista externa. Techo del paladar plano; forma un ángulo recto con las paredes alveolares. Todos los molariformes trilobulados (menos evidente en el M1). Labios de la cresta deltopectoral del húmero casi rectos, formando una "V" casi perfecta, a diferencia de $P$. subintermedius Castellanos,1937, en la cual el labio póstero-externo es convexo y dirigido hacia afuera. Fémur más grácil que en $P$. subintermedius y de robustez parecida a P. greslebini Castellanos, 1942. Coraza elevada y comprimida. Osteodermos de superficie poligonal, con figuritas poligonales pequeñas (tubérculos), de superficie plana y tamaño similar, distribuidas por toda la superficie, sin patrón de ordenamiento. Sin figura central (excepto en los osteodermos cercanos a los márgenes), al igual que en todas las especies del género, salvo $P$. intermedius Lydekker, 1895, y $P$. jaguaribensis Moreira, 1965. Tubo caudal cilíndrico en su región proximal, luego fuertemente deprimido. Región distal del dorso del tubo con dos grandes depresiones subelípticas rugosas, una de cada lado; por detrás de ellas, dos depresiones más pequeñas y subcirculares (sólo una en $P$. subintermedius y $P$. greslebini), no simétricas (simétricas en $P$. jaguaribensis). Lados del tubo con una depresión rugosa subcircular proximal, seguida por cuatro figuras subelípticas rugosas más grandes y deprimidas, con una elevación central.

Diagnosis. Skull highly vaulted, nasal bones obliquely descending anteriorly; pneumatization degree more important than in Panochthus frenzelianus Ameghino, 1889, and even more important than in Panochthus sp. from Ensenadan of Argentina. Orbit and temporal fossa separated by a complete postorbital process. Palate flat, forming a right angle with the alveolar processes. Molars trilobated (less evident in M1). Lips of the deltopectoral crest of humerus almost straight, forming an almost perfect " $V$ ", differently from P. subintermedius Castellanos, 1937, where the posteroexternal lip is convex and directed laterally. Femur much slender than in P. subintermedius, with a robustness similar to P. greslebini Castellanos, 1942. Armor deep and compressed. Osteoderms of
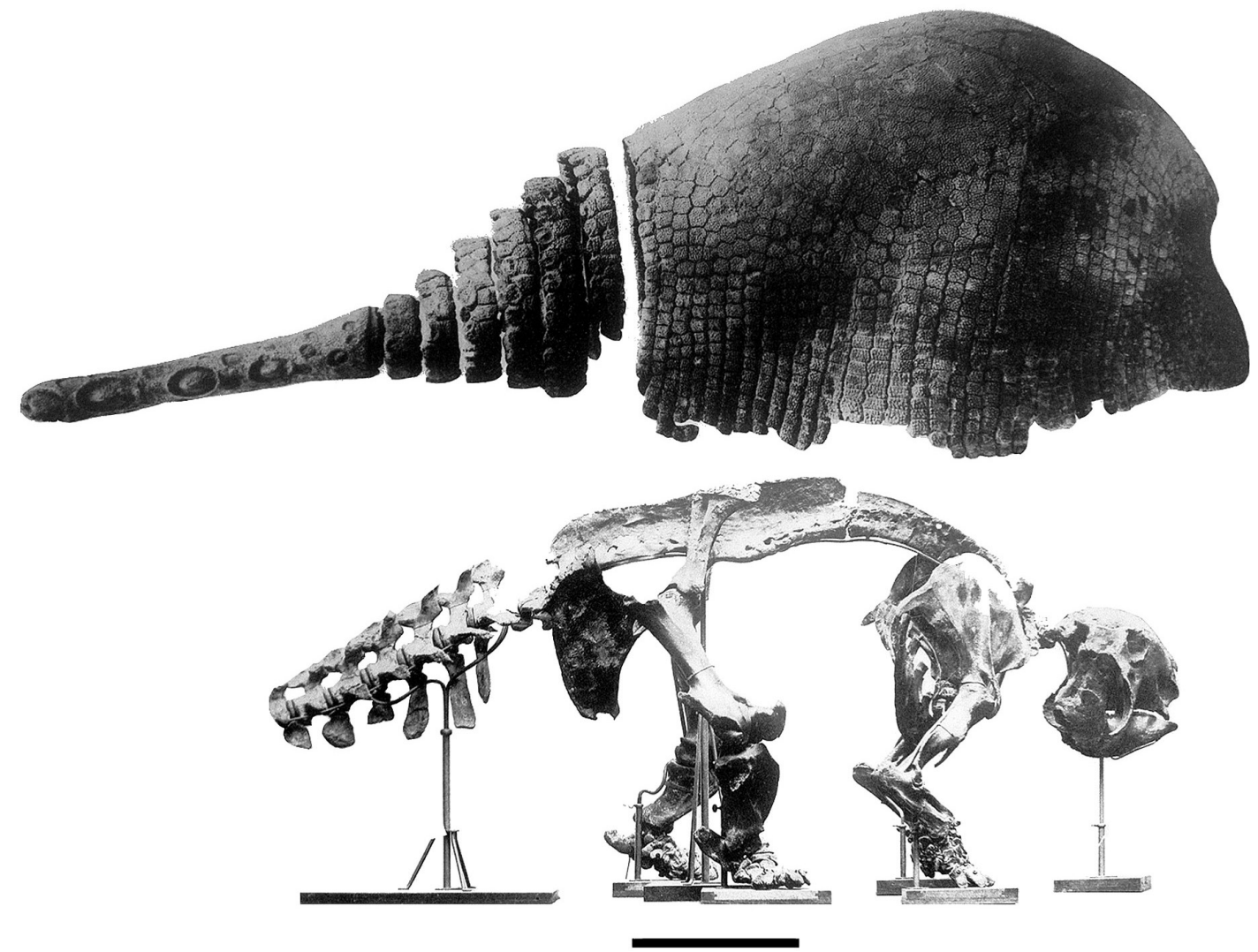

Figura 2. Vista general de la coraza y el esqueleto (MLP 16-29), armado bajo la dirección de F. Ameghino. Reproducido y levemente modificado de Lydekker (1895: láms. 22, 23). Escala $=50 \mathrm{~cm}$.

Figure 2. General view of of the carapace and the skeleton (MLP 16-29), mounted under the direction of F. Ameghino. Reproduced and slightly modified from Lydekker (1895, plates 22, 23). Scale bar $=50 \mathrm{~cm}$. 
polygonal surface, with small polygonal figures (tubercles), flat and similar size, with not ordered patterning. Absence of central figure, except from marginal osteoderms), as in all species of the genus, except from P. intermedius Lydekker, 1895, and P. jaguaribensis Moreira, 1965. Caudal tube cylindrical proximally, afterwards strongly depressed. Distal dorsal region of tube with two large depressions subeliptical and rugose, one of each side. Behind them, two depressions smaller and subcircular (only one in P. subintermedius and P. greslebini), not symmetrical (symmetrical in P. jaguaribensis). The sides of the tube with a rugose depression subcircular proximal, followed by four large rugose subeliptical figures, depressed and with a central elevation.

Descripción comparada. Cráneo: la conformación y dimensión es similar a la del cráneo de Panochthus frenzelianus, características que son notoriamente diferentes a las que presenta el único cráneo del

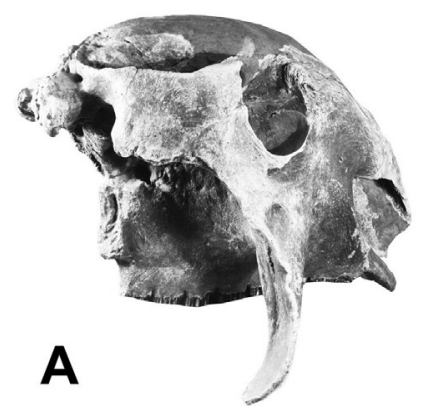

Ensenadense hasta ahora conocido (Panochthus sp., véase Zamorano \& Scillato-Yané, 2006; Zamorano, 2009), el cual es evidentemente menos neumatizado y de tamaño superior. En vista anterior, los nasales son descendentes, como ocurre en los cráneos de todas las especies de Panochthus y la zona naso-frontal se inclina hacia abajo. En esta última la neumatización posee mayor grado que en $P$. frenzelianus. La escotadura orbitaria es semicircular, como en $P$. frenzelianus, a diferencia del Panochthus sp. del Ensenadense (cráneo MLP 84-IX-2-11, en adelante Panochthus sp. E) es alargada. La fosa orbitaria y la fosa temporal están separadas en la vista externa por un proceso postorbital completo. En P. frenzelianus y en Panochthus sp. E, a semejanza de Glyptodon, dicho proceso postorbital es incompleto, de manera que las fosas orbitaria y temporal se comunican en vista externa. En el contorno de la fosa orbitaria el tubérculo lacrimal sobresale, a diferencia

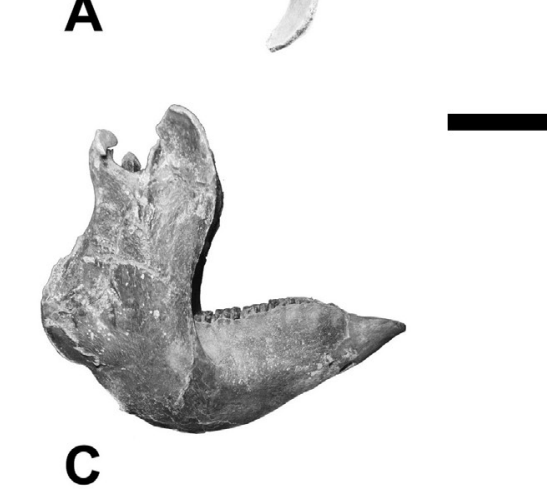

B
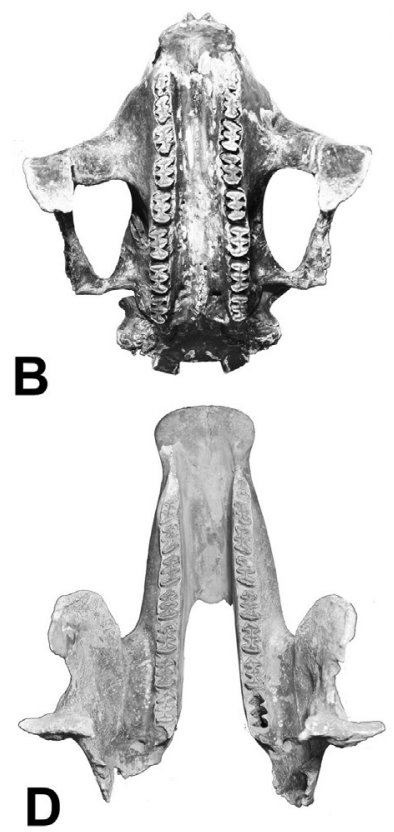

C
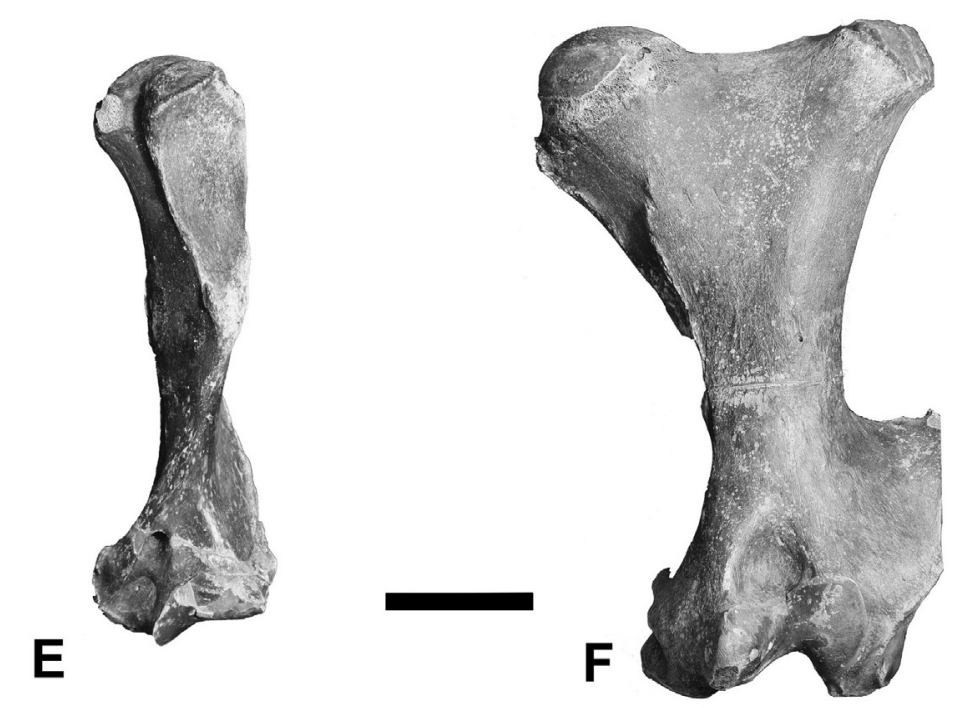

Figura 3. Esqueleto MLP 16-29. Cráneo: A, vista lateral; B, vista ventral. Mandíbula: C, vista lateral; D, vista dorsal. Húmero izquierdo: E, vista anterior. Fémur izquierdo: $\mathbf{F}$, vista anterior. Escalas $=100 \mathrm{~mm}$.

Figure 3. Skeleton MLP 16-29. Skull: A, lateral view; B, ventral view. Mandible: C, lateral view; D, dorsal view. Left humerus: E, anterior view. Left femur: F, anterior view. Scale bars $=100 \mathrm{~mm}$. 
de P. frenzelianus. La apófisis descendente del maxilar y el foramen infraorbitario tiene tamaños y formas similares a $P$. frenzelianus. En vista lateral, el arco cigomático se ensancha debajo de la fosa temporal, a diferencia del arco de P. frenzelianus y Panochthus sp. E, en los cuales el ancho del arco se mantiene más constante. En cuanto a la robustez del arco, ésta es mayor en Panochthus sp. E que la de los Panochthus registrados para el lapso Bonaerense-Lujanense. A diferencia de P. frenzelianus y de Panochthus sp. E, el arco cigomático sobresale lateralmente. El arco cigomático, por detrás de la órbita y hasta poco antes de su inserción, es horizontalmente recto, a diferencia de P. frenzelianus y Panochthus sp. E, en los cuales es curvo. Los huesos frontales y nasales se expanden lateralmente. En vista dorsal, el cráneo es muy abovedado. Lamentablemente en esta vista no se puede comparar con $P$. frenzelianus, el único cráneo conocido se encuentra exhibido en el American Museum of Natural History (AMNH 11243), no se le puede quitar el escudete cefálico para observar esta zona. En vista posterior, los dos cóndilos tienen forma subcuadrada y delimitan el foramen magnum, el cual es elíptico, con el diámetro horizontal mayor. El contorno de la abertura de las coanas es rectangular, con el diámetro vertical mayor. En vista ventral, el techo del paladar es plano, a diferencia de Panochthus sp E, en el cual es cóncavo transversalmente. Dicho techo se une a las paredes alveolares abruptamente, formando un ángulo recto; en cambio, en Panochthus sp. E, la unión es gradual y el ángulo resultante es obtuso. De esta manera, el techo del paladar, rodeado por paredes alveolares rectas, presenta el aspecto de una canaleta de sección rectangular.

Mandíbula: las características del hueso mandibular/ dentario son semejantes a las de las otras especies del género. Es robusto, similar al de las más grandes especies de Glyptodon. Las ramas ascendentes, en vista lateral, ocultan los dos molariformes más posteriores. Estas ramas se encuentran más inclinadas hacia la zona anterior a nivel de las apófisis coronoides; dichas apófisis alcanzan la misma altura que los respectivos cóndilos mandibulares. En vista lateral también se observa que el borde alveolar presenta una forma de "S" apaisada, la cual alcanza su máxima altura a nivel del tercer molariforme. La escotadura sigmoidea es más amplia y más profunda que la de Glyptodon y levemente más profunda que la de Neosclerocalyptus.

Dentición: la serie dentaria superior está compuesta por ocho molariformes, todos trilobulados y con sus lóbulos alineados y de dimensiones similares, a diferencia del Panochthus sp. E, en el cual el M1 es de sección subelíptica. En los tres primeros molariformes de MLP 84-IX-2-11, los lóbulos se disponen oblicuamente a la serie dentaria, con una orientación antero-lingual a póstero-labial. En lo que respecta a la serie dentaria inferior, el $\mathrm{m} 1$ es trilobulado labialmente y redondeado, sin surcos longitudinales lingualmente, en tanto que los $\mathrm{m} 2-\mathrm{m} 8$ son totalmente trilobulados.

Esqueleto postcraneal: el esqueleto del ejemplar MLP 16-29 está completo. Hay muchos elementos del esqueleto postcraneal que en otras especies de Panochthus no se conocen, por lo cual aquí solamente se describirán comparativamente húmero y fémur, con los cuales el cotejo sí es posible. Asimismo ver Tabla 2.

Húmero: tamaño menor que el de Panochthus subintermedius. El diámetro trasverso y el antero-posterior de la cabeza humeral son similares. En cambio, en P. subintermedius el diámetro trasverso es mayor al antero-posterior. La cabeza humeral de Panochthus no está circunscrita por un cuello bien acentuado. La corredera bicipital está bien marcada, aunque no es tan profunda como en Glyptodon. En P. tuberculatus, la cresta deltopectoral está muy desarrollada y sus labios son casi rectos, formando una " $\mathrm{V}$ " casi perfecta (con el vértice orientado distalmente), a diferencia de P. subintermedius, en la cual su labio póstero-externo es convexo y dirigido hacia afuera. La superficie de la cresta deltopectoral es lisa en todas las especies del género; es ligeramente cóncava en $P$. tuberculatus, pero lo es más pronunciadamente en $P$. subintermedius. Por debajo de la cresta deltopectoral, la diáfisis presenta un aspecto cónico que se va aplanando hacia la epífisis distal. Dicha epífisis presenta el foramen entepicondiliano, como en Neosclerocalyptus,

Tabla 2. Medidas (en cm) del neotipo (MLP 16-29), comparadas con las de P. subintermedius (MACN-Pv 5130) and P. greslebini (MNRJ 2760).

Table 2. Measurements (in cm) of the neotype (MLP 16-29) compared with $P$. subintermedius (MACN-Pv 5130) and $P$. greslebini (MNRJ 2760).

\begin{tabular}{|c|c|c|c|}
\hline $\begin{array}{c}\text { Huesos } \\
\text { postcraneales / } \\
\text { Medidas }\end{array}$ & $\begin{array}{c}\text { P. tuberculatus } \\
\text { Neotipo: MLP 16-29 }\end{array}$ & $\begin{array}{l}\text { P. subintermedius } \\
\text { MACN-Pv } 5130\end{array}$ & $\begin{array}{l}\text { P. greslebini } \\
\text { MNRJ } 2760\end{array}$ \\
\hline \multicolumn{4}{|l|}{ Húmero } \\
\hline Largo & 37 & 38 & \\
\hline Ancho mínimo & 4,5 & 6 & \\
\hline \multicolumn{4}{|l|}{ Radio } \\
\hline Largo & 16 & & \\
\hline Ancho mínimo & 3 & & \\
\hline \multicolumn{4}{|l|}{ Ulna } \\
\hline Largo & 27 & & \\
\hline Ancho mínimo & 6,5 & & \\
\hline \multicolumn{4}{|l|}{ Fémur } \\
\hline Largo & 49 & 54,3 & 53,4 \\
\hline Ancho mínimo & 10,1 & 11,5 & 9 \\
\hline \multicolumn{4}{|l|}{ Tibia-fíbula } \\
\hline Largo & 30,6 & & \\
\hline Ancho mínimo & Tibia: 10 Fíbula: 9 & & \\
\hline \multicolumn{4}{|l|}{ Escápula } \\
\hline Diám. transvero & 49,5 & & \\
\hline \multicolumn{4}{|l|}{ Curva del tubo } \\
\hline dorsolumbar & 159 & & \\
\hline
\end{tabular}


Doedicurus y la mayoría de los géneros de gliptodóntidos, y a diferencia, por ejemplo, de Glyptodon. Este último foramen está ubicado en la zona antero-ventral de la epífisis y tiene un contorno subcircular. Inmediatamente por debajo y más hacia el centro, existe una depresión subcircular, la cavidad coronoides, la que, como en los mencionados gliptodóntidos que poseen foramen entepicondiliano, no es muy profunda, a diferencia de Glyptodon, en el cual la cavidad coronoides es casi tan profunda como la cavidad olecraneana. En la cara posterior del húmero, a nivel de la epífisis distal, se aprecia que la cavidad olecraneana es amplia y está bien excavada, aunque no tanto como en Glyptodon. Presenta una forma subtriangular con su eje mayor ubicado en sentido transverso.

Fémur: es ligeramente más grácil que el de Panochthus subintermedius, de robustez similar al de $P$. greslebini. En las epífisis, como en los demás Glyptodontidae, presenta un mayor desarrollo transverso que antero-posterior. En la epífisis proximal, la superficie articular de la cabeza femoral presenta forma subcircular, como en P. greslebini y P. subintermedius. En el sector externo de esta epífisis se encuentra el trocánter mayor cuya superficie, adquiere forma más laminar que en $P$. greslebini. El trocánter mayor está ubicado, al igual que en $P$. greslebini y a diferencia de Neosclerocalyptus y Propalaehoplophorus, en un plano ligeramente más elevado respecto de la cabeza femoral. Separando ambas estructuras no se aprecia (sobre el borde superior) una concavidad marcada, como sí se ve en $P$. subinteredius. La superficie del hueso (en esta separación) es plana en Panochthus. En la cara anterior se observa que, al igual que en $P$. greslebini, la depresión triangular ubicada por debajo de la epífisis proximal es levemente más profunda que en $P$. subintermedius. La zona más distal de la diáfisis es de sección subcircular; aquí el hueso exhibe el menor diámetro. A este nivel, e inmediatamente por arriba de la epífisis distal, se encuentra, en el borde externo, el tercer trocánter, de forma subtriangular, con su vértice ligeramente inclinado hacia adelante; toda su morfología es muy similar

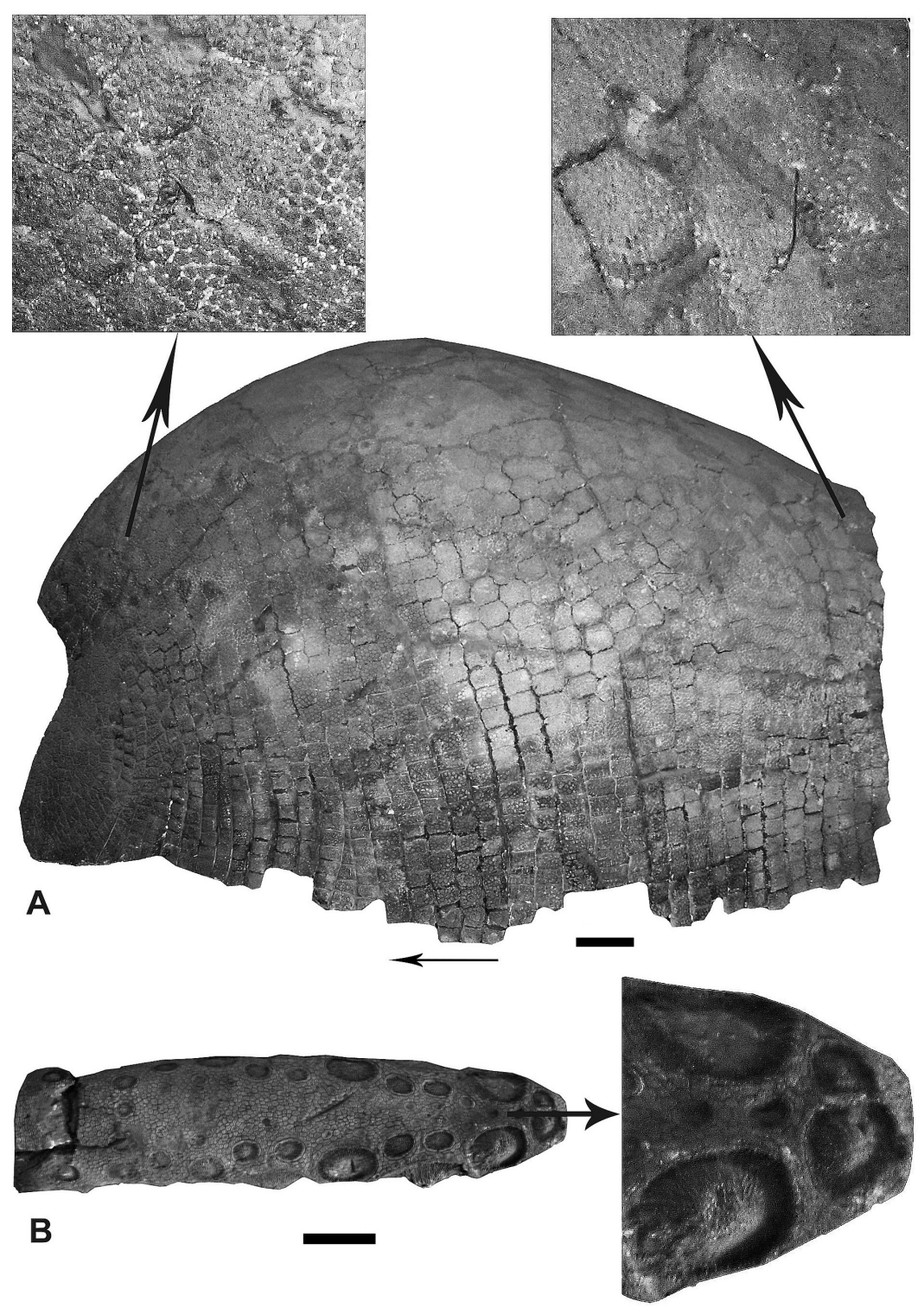

Figura 4. MLP 16-29. A, coraza dorsal; B, tubo caudal. Escala $=100 \mathrm{~mm}$.

Figure 4. MLP 16-29. A, dorsal carapace; B, caudal tube. Scale bar $=100 \mathrm{~mm}$. 
a la de Neosclerocalyptus. En la epífisis distal se observan los cóndilos medial y lateral y, entre ambos, la tróclea femoral. Inmediatamente por encima de esta tróclea está el hueco supratroclear, muy evidente en las especies de este género. El cóndilo medial está situado en un plano más bajo que el lateral. La cara posterior del fémur en este género es plana y su superficie es bastante lisa. En la epífisis proximal, se observa cómo la cabeza femoral y el trocánter mayor sobresalen lateralmente. La diáfisis es más plana que en la cara anterior, sin accidentes de relevancia. En vista posterior, en la epífisis distal, los cóndilos son las dos zonas más conspicuas. En la epífisis proximal, se observa cómo la cabeza femoral y el trocánter mayor sobresalen lateralmente. La diáfisis es más plana que en la cara anterior, sin accidentes de relevancia; sobre todo, se puede apreciar una región plana rectangular que ocupa unos dos tercios del ancho de la diáfisis, delimitada por los márgenes del tercer trocánter que se une al epicóndilo externo. En otros gliptodóntidos, como Propalaehoplophorus australis Ameghino, 1887, el tercer trocánter es más elevado y no se une al epicóndilo externo, de manera que no aparece la zona rectangular plana mencionada. En vista posterior, en la epífisis distal, los cóndilos son los dos elementos más proyectados que se pueden apreciar en el fémur.

Coraza dorsal: Su tamaño es semejante al de Panochthus frenzelianus y menor que el de $P$. intermedius. Aunque la coraza de $P$. subintermedius no está completa, esta última parecería ser similar o levemente mayor que la de $P$. tuberculatus (Cruz et al., 2007, 2011). La coraza es más elevada de perfil y más comprimida lateralmente que la de $P$. frenzelianus y $P$. intermedius, semejante a la de $P$. subintermedius. Los osteodermos son poligonales, tendiendo a ser hexagonales en la zona dorsal y cuadrangulares en los laterales (en este sector, casi rectangulares) y en la zona cercana a las escotaduras anterior y posterior. La ornamentación, en toda la coraza excepto en los márgenes, consiste en figuritas poligonales pequeñas (tubérculos), de superficie plana y similares en tamaño entre sí, distribuidas por toda la superficie, sin que aparentemente exista algún patrón de ordenamiento; lo mismo ocurre en $P$. frenzelianus y P. subintermedius; en cambio, en el único fragmento de coraza conservado (región dorsal según Moreira, 1971 y Porpino \& Bergqvist, 2002) de P. jaguaribensis, y en las regiones antero-dorsal y póstero-dorsal de $P$. intermedius, los osteodermos presentan una figura central distinguible. Lamentablemente el ejemplar que se describe (MLP 16-29) tiene los bordes laterales rotos. En P. tuberculatus, según Burmeister (1874), en estos bordes se observa en las cuatro hileras de osteodermos más cercanos al borde una notable figura central rodeada por figuritas periféricas; en la coraza de P. frenzelianus, la cual está preservada completamente, también se observan estas cuatro hileras de osteodermos con figura central. En MLP 16-29 solamente se aprecia dicha figura central en los osteodermos de algunas zonas en las cuales el borde se preserva parcialmente. Cabe señalar aquí que dicha zona de la coraza de gliptodontes (bordes de los márgenes laterales) son las menos preservadas, ya que se rompen con más facilidad.

Anillos caudales: el neotipo de Panochthus tuberculatus posee seis anillos, el más distal articulado con el tubo caudal. Cada anillo está formado por dos series de osteodermos. La ornamentación de estos últimos es similar a los de la coraza dorsal. Por desgracia en ninguna otra especie perteneciente al género se han conservado estos anillos.

Tubo caudal: presenta un tamaño y una morfología similar al de las especies del género conocidas para el lapso Bonaerense-Lujanense, es decir cilíndrico-cónico y disminuyendo su diámetro dorso-ventral en sentido distal, mientras que la extremidad proximal es de contorno casi completamente circular; el largo es bastante menor que el de Panochthus subintermedius; el tubo de P. subintermedius es menos deprimido y su extremo distal no es romo, como lo es en $P$. tuberculatus, sino que finaliza en punta. Las figuritas que cubren la superficie del tubo son semejantes a las de la coraza dorsal. En vista dorsal se observan, desde la región proximal, depresiones circulares rugosas, cercanas a ambos márgenes laterales (figuras marginales), mucho más profundas que en $P$. jaguaribensis y más, aunque no tanto, que en $P$. greslebini, especies en las cuales además se encuentran en menor cantidad. En P. tuberculatus, la profundidad y cantidad de estas depresiones circulares rugosas es semejante a $P$. frenzelianus, a diferencia de $P$. subintermedius, en la cual son más grandes y se encuentran aún más cercanas a los bordes laterales. Desde esta vista, distalmente se distinguen dos grandes depresiones subelípticas rugosas, ubicadas simétricamente una de cada lado, las cuales logran apreciarse desde la vista lateral (figuras dorsales). Estás dos grandes depresiones subelípticas rugosas se presentan en todas las especies de Panochthus. En el extremo distal, distalmente a las grandes depresiones recién mencionadas, hay dos depresiones más pequeñas y subcirculares (figuras apicales), no simétricas; en P. jaguaribensis éstas son simétricas; en P. greslebini y P. subintermedius en este lugar hay una sola depresión, la cual está ubicada en el centro y posee un diámetro mayor. En vista ventral, tanto en el material que aquí se describe como en todos los conocidos para el género, se observa un diseño similar al de la cara dorsal, aunque con las figuritas y las rugosidades menos marcadas. En vista lateral existe una depresión rugosa y subcircular seguida, hacia el extremo distal, por cuatro figuras subelípticas rugosas más grandes, deprimidas y elevadas en el centro; éstas se encuentran rodeadas por varias hileras de figuritas similares a las de la cara dorsal. En P. frenzelianus y $P$. greslebini también hay cuatro, en P. jaguaribensis hay cinco figuras subelípticas a continuación de la mencionada depresión subcircular, aunque una de ellas corresponde al par dorsal-ventral (ver Porpino \& Bergqvist, 2002); P. subintermedius presenta seis figuras laterales en la porción izquierda y cinco en la porción derecha. Cabe mencionar que en publicaciones en las que han participado dos de los autores [M.Z. y G.J.S.-Y.] (e.g. Cruz et al., 2011) y en otras contribuciones previas (e.g. Castellanos, 1942; Moreira, 1971; Porpino \& Berqvist, 2002), la figura lateral más distal es mencionada como figura terminal. De los elementos anatómicos descriptos del ejemplar MLP 16-29 es sin duda el tubo caudal el más variable dentro de la especie.

\section{DISCUSIÓN}

El ejemplar de Panochthus tuberculatus MLP 16-29 cumple con las condiciones exigidas por el Código Internacional 
de Nomenclatura Zoológica vigente (ICZN, 1999, Art. 75.3). Además, como ya se dijo, no se corresponde a las "Circunstancias excluidas" (ICNZ, 1999, Art. 75.2). La designación de un neotipo es válida sólo cuando en la publicación queden cumplimentados los incisos siguientes:

* 75.3.1. Declaración de que se designa con el propósito expreso de clarificar la posición taxonómica. En este caso la designación de neotipo, al ser el material tipo de la especie tipo del género, es imprescindible para realizar comparaciones y así poder tomar decisiones sobre cuáles de las especies incluidas en el género pueden considerarse validas y cuáles no.

*75.3.2. Declaración de los caracteres que el autor considera distintivos o una referencia bibliográfica a dicha declaración. Se mencionan en "Sistemática paleontológica".

*75.3.3. Datos y descripción para asegurar el reconocimiento del ejemplar designado. Se mencionan en "Sistemática paleontológica".

*75.3.4. Razones del autor para considerar el material tipo perdido o destruido. Se mencionan en "Introducción".

*75.3.5. Evidencia de que el neotipo concuerda con lo que se sabe del material tipo original. En este caso se ajusta a la descripción brindada por Owen (1845: 120, lám. 5, figs. 3-5). Dicha descripción es parcialmente similar a las realizadas por Ameghino (1889:834) y Castellanos (1942: 534, fig. 216), en ambos casos para Panochthus morenoi Ameghino, 1889, non Ameghino (1881a), considerada en este trabajo como un sinónimo de P. tuberculatus.

* 75.3.6. Evidencia de que el neotipo proviene de tan cerca como sea factible de la localidad tipo original [Art.76.1] y del mismo horizonte geológico [véase también el Art. 76.3 y la Recomendación 76.01]. Del material original tipo no se conoce otra procedencia que Pleistoceno de la Provincia de Buenos Aires; el ejemplar de Tapalqué (MLP 16-29) cumple con estas condiciones del Código.

* 75.3.7. Declaración de que el neotipo es propiedad de una institución científica reconocida y que tenga una colección con la infraestructura adecuada para conservar al neotipo. El ejemplar se halla en el Museo de La Plata (Universidad Nacional de La Plata) desde hace más de 110 años (véase Lydekker, 1895; Castellanos, 1942). El Departamento Científico Paleontología Vertebrados del Museo de La Plata posee la más completa colección de fauna cuaternaria de la región pampeana del mundo.

El neotipo aquí designado, basado en un material muy completo y muy bien preservado, permite comparar la especie tipo con otras especies del género reconocidas sólo a base de algunas estructuras anatómicas (e.g. tubo caudal).

\section{CONCLUSIONES}

El ejemplar MLP 16-29, consistente en el esqueleto y la coraza casi completos de un gliptodóntido, hasta ahora atribuido a Panochthus morenoi, es designado neotipo de $P$. tuberculatus. De acuerdo al análisis efectuado, $P$. morenoi (sensu Ameghino, 1889), resulta un sinónimo de $P$. tuberculatus. La localidad tipo de P. tuberculatus es Tapalqué, partido de Tapalqué, centro de la Provincia de Buenos Aires,
Argentina. Finalmente, el estudio comparativo del neotipo permite reconocer una combinación exclusiva de caracteres anatómicos, los cuales reafirman la validez de la especie $P$. tuberculatus.

\section{AGRADECIMIENTOS}

Al E. Soibelzon (MLP), por su ayuda en la toma de medidas del material en exhibición en el MLP, de no muy fácil acceso en este caso, así como por la obtención de gran parte de las fotografías; F. Góis (MLP), por su auxilio en la toma de algunas otras fotografías y medidas; J.C. Fernicola (MACN), por su asesoramiento en diversos aspectos de este trabajo, así como por su amable disposición para informarnos de cuestiones relativas a las colecciones del MACN; D. Brandoni, por sus sugerencias relativas a la confección del mapa de distribución y comentarios paleobiogeográficos; M.A. Reguero y L.H. Pomi, por su cordialidad al facilitar la consulta de las colecciones del MLP; a los revisores, por sus valiosos aportes que permitieron mejorar este trabajo.

\section{REFERENCIAS}

Ameghino, F. 1881a. La Formación Pampeana ó estudio sobre los terrenos de transporte de la cuenca del Plata. Paris y Buenos Aires, G. Masson/Igon Hermanos, 376 p.

Ameghino, F. 1881b. La antigüedad del hombre en el Plata. París y Buenos Aires, G. Masson-Igon Hermanos, 2:1-557.

Ameghino, F. 1889. Contribución al conocimiento de los mamíferos fósiles de la República Argentina. Actas Academia Nacional de Ciencias en Córdoba, 6:1-1027.

Ameghino, F. 1895. Sur les édentés fósiles de l'Argentine. Examen critique, revisión et correction de l'ouvrage de Mr. R. Lydekker: "The extinct edentates of Argentina", etc. Revista del Jardin Zoológico de Buenos Ayres, 3:97-192.

Ameghino, F. 1898. Sinopsis geológico-paleontológica de la Argentna. In: $2^{\circ}$ Censo de la República Argentina, Territorio, 1:115-228.

Ameghino, F. 1920. Sur les édentés fossiles de l'Argentine. Examen critique, révision et correction de l'ouvrage de M. R. Lydekker "The extinct edentates of Argentina", etc. (Ouvrage à démi-inédite avec des notes additionelles sur quelques ongulés et carnassiers)/ Sobre los desdentados fósiles de la Argentina. Exámen crítico, revisión y corrección de la obra del Señor R. Lydekker "The extinct edentates of Argentina", etc. (Obra mitad inédita y con notas adicionales sobre algunos ungulados y carniceros). In: Obras Completas y Correspondencia Cientifica de Florentino Ameghino, Taller de Impresiones Oficiales, La Plata, 11:447-909.

Bergqvist, L.P. 1993. Jazimentos pleistocênicos do Estado da Paraíba e seus fósseis. Revista Nordestina de Biologia, 8:143-158.

Burmeister, G. 1866. Lista de los mamíferos fósiles del terreno diluviano. Anales del Museo Público de Buenos Aires, 1:121-232.

Burmeister, H. 1867. Verzeichniss der fossilen Säugethiere im Diluvium Südamerika. Zeitschrift für die Gesammten Naturwissenschaften, 30:528-529.

Burmeister, G. 1874. Monografía de los glyptodontes en el Museo Público de Buenos Aires. Anales del Museo Público de Buenos Aires, 2:355-412.

Burmeister, H. 1872. Uebersicht der Glyptodonten. Archiv für Naturgeschichte, 38:250-264.

Castellanos, A. 1925. Breves notas sobre evolución de la coraza y del extremo caudal en los Loricata (= Hicanodonta)". Revista de "El Circulo", número extraordinario, p. 93-96.

Castellanos, A. 1933. Las arenas del antiguo Río Paraná y la perforación de Villa Ballester (F.C.C.A)" Quid novi?. Revista 
de Asociaciones de Ex-Alumnas y Padres de la Escuela Normal, $\mathrm{n}^{\mathrm{o}} 2$, año II, $\mathrm{n}^{\mathrm{o}} 5$, p. 11 del separado.

Castellanos, A. 1937. Los sedimentos del Pampeano inferior y del Araucano en el Valle de Los Reartes (Sierra de Córdoba). Publicaciones de la Facultad de Ciencias Matemáticas, FísicoQuímicas y Naturales aplicadas a la Industria, 6:1-110.

Castellanos, A. 1942. A propósito de los géneros Plohophorus, Nopachthus y Panochthus (3a. parte). Publicaciones del Instituto de Fisiografía y Geología, 3:413-592.

Cruz, L.E.; Zamorano, M. \& Scillato-Yané, G.J. 2007. Panochthus subintermedius Castellanos (Xenarthra, Glyptodontidae): redescripción e implicancia biocronológica. Ameghiniana, 44:13R.

Cruz, L.E.; Zamorano, M. \& Scillato-Yané, G.J. 2011. Diagnosis and redescription of Panochthus subintermedius Castellanos (Xenarthra, Glyptodontia) from the Ensenadan (early-middle Pleistocene) of Buenos Aires (Argentina). Paläontologische Zeitschrift, 85:115-123. doi: 10.1007/s12542-010-0084-7

Fariña, R.A. 2000. Física y Matemáticas para reconstruir la vida en el pasado. Actas de Fisiología, 6:45-70.

Figini, A.J.; Huarte, R.; Carbonari, J. \& Tonni, E.P. 1998. Edades $\mathrm{C}^{14}$ en un perfil del arroyo Tapalqué, provincia de Buenos Aires, Argentina. Contribución a la cronología de los acontecimientos faunístico ambientales. In: CONGRESO LATINOAMERICANO DE GEOLOGÍA, 10, 1998. Actas, Buenos Aires, 1:27-31.

Gilette, D.D. \& Ray, C.E. 1981. Glyptodonts of North America. Smithsonian Contributions to Paleobiology, 40:1-255.

Hoffstetter, R. 1963. Les glyptodontes du Pléistocène de Tarija (Bolivie). 1: Genres Hoplophorus et Panochthus: Comptes Rendus de la Société Géologique de France, 1963:96.

Hoffstetter, R. 1964. Les glyptodontes du Pléistocène de Tarija (Bolivie). 1: Genres Hoplophorus et Panochthus. Bulletin de la Société Géologique de France, 5:126-133.

Hoffstetter, R. 1978. Une faune de mammifères pléistocènes au Paraguay. Comptes Rendus de la Société Géologique de France, 1978:32-33.

International Commission on Zoological Nomenclature. 1999. International Code of Zoological Nomenclature. International Trust for Zoological Nomenclature, London, p. 1-156.

Kerber, L. \& Oliveira, E.V. 2008. Novos fósseis de vertebrados para a Sanga da Cruz (Pleistoceno Superior), Alegrete, RS, Brasil. Revista Pesquisas em Geociências, 35:39-45.

Lydekker, R. 1887. Catalogue of the fossil Mammalia in the British Museum (Natural History). London, British Museum (Natural History), 5:1-345.

Lydekker, R. 1895. Contributions to the knowledge of the fossil vertebrates of Argentina, II. 2. The extinct edentates of Argentina/ Contribuciones al conocimiento de los vertebrados fósiles de la Argentina, Los desdentados extinguidos de la Argentina. Anales del Museo de La Plata (Paleontología Argentina), 3:1-118.

Mones, A. 1986. Palaeovertebrata Sudamericana. Catálogo Sistemático de los Vertebrados Fósiles. Parte I (Lista preliminar y bibliografía). Courier Forschungsinstitut Senckenberg, 82:1-625.

Mones, A. 1994. Las vicisitudes del género Panochthus Burrmeister, 1866 (Mammalia: Cingulata: Glyptodontidae). Comunicaciones Paleontológicas del Museo de Historia Natural de Montevideo, 2:79-86.

Mones, A. \& Francis, J.C. 1973. Lista de los vertebrados fósiles del Uruguay, II. Mammalia. Comunicaciones Paleontológicas del Museo de Historia Natural de Montevideo, 1:39-97.

Moreira, L.E. 1965. Notas prévias sobre nova espécie de mamífero fóssil do Estado do Ceará, Brasil. Hy Hy Té, Revista da Faculdade de Filosofia do Crato, 2:41-49.

Moreira, L.E. 1971. Os gliptodontes do Nordeste do Brasil. Anais da Academia Brasileira de Ciencias, 43:529-552.

Moreno, F.P. 1888. Informe preliminar de los progresos del Museo La Plata durante el primer semestre de 1888, presentado al señor ministro de Obras Públicas de la provincia de Buenos Aires. Boletín del Museo La Plata, p. 1-35.
Nodot, L. 1855. Description d' un nouveau genre d' édenté fossile renfermant plusieurs espèces voisines des glyptodons, et classification méthodique de treize espèces appartenant à deux genres. Comptes Rendus de l'Académie des Sciences, 41:335-338.

Nodot, L. 1957. Description d'un nouveau genre d'edenté fossile renformat plusierus especes voisines du Glyptodon. Mémoire Academie Impériale Sciences, Arts, Belles Lettres Dijon, 5:1-170.

Owen, R. 1839. Description of a tooth and part of the skeleton of the Glyptodon, a large quadruped of the edentate order, to which belongs the tessellated bony armour figured by Mr. Clift in his memoir on the Megatherium, brought to England by Sir Woodbine Parish, F.G.S. Proceedings of the Geological Society of London, 3:108-113.

Owen, R. 1845. Descriptive and illustrated catalogue of the fossil organic remains of Mammalia and Aves contained in the Museum of the Royal College of Surgeons of England. R. \& J. E. Taylor, London, p. 1-391.

Paula Couto, C. 1954. Sobre alguns mamíferos fósseis do Ceará Arquivos do Museu Nacional, 42:195-210.

Porpino, K.O. \& Bergqvist, L.P. 2002. Novos achados de Panochthus (Mammalia, Cingulata, Glyptodontoidea) no Nordeste do Brasil. Revista Brasileira de Paleontologia, 4:51-62.

Scalabrini, P. 1887. Cartas cientificas al General Eduardo Racedo Paraná, Museo de la Provincia de Entre Ríos, Tipografía y Encuadernación La Velocidad, p. 1-209.

Scillato-Yané, G.J.; Carlini, A.A.; Vizcaíno, S.F. \& Ortiz-Jaureguizar, E. 1995. Xenarthra. In: M.T. Alberdi; G. Leone \& E.P. Tonni (eds.) Evolución biológica y climática de la región Pampeana durante los últimos cinco millones de años. Un ensayo de correlación con el Mediterráneo Occidental. Monografias del Museo de Ciencias Naturales, Consejo Superior de Investigaciones Científicas, 12:183-209.

Sisson, S. \& Grossman, J.D. 1959. Anatomía de los animales domésticos. Barcelona, Salvat Editores, 952 p.

Tauber, A.A. \& Palacios, M.E. 2007. Nuevos registros de mamíferos cuaternarios de gran porte en la provincia de Santa Cruz, República Argentina. Ameghiniana, 44:41R.

Tonni, E.P.; Soibelzon, E.; Cione, A.L.; Carlini, A.A.; Scillato Yané, G.J.; Zurita, A.E. \& Paredes Ríos, F. 2009. Preliminar correlation of the Pleistocene sequences of the Tarija valley (Bolivia) with the Pampean chronological standard. Quaternary International, 210:57-65. doi:10.1016/j.quaint.2009.06.015

Zamorano, M. 2009. Estudio de un cráneo de Panochthus del Ensenadense (Pleistoceno temprano-medio) comparado con otros del mismo género del Bonaerense y Lujanense (Pleistoceno medio-Holoceno temprano), mediante análisis por componentes principales. Ameghiniana, 46:97R.

Zamorano, M. \& Scillato-Yané, G.J. 2006. Descripción comparativa del cráneo de Panochthus cf. intermedius Lydekker (Glyptodontidae, Hoplophorinae, Panochthini), del Ensenadense de la región pampeana. Ameghiniana, 43:58-59.

Zamorano, M.; Scillato-Yané, G.J.; González Ruiz, L.R. \& Zurita, A.E. 2011. Revisión de los géneros Nopachtus Ameghino y Phlyctaenopyga Cabrera (Xenarthra, Glyptodontidae, Hoplophorinae) del Mioceno tardío y Plioceno de Argentina. Revista del Museo Argentino de Ciencias Naturales "Bernardino Rivadavia", 13:59-68.

Zurita,A.E. 2007. Sistemática y evolución de los Hoplophorini (Xenarthra, Glyptodontidae, Hoplophorinae. Mioceno tardioHoloceno temprano). Importancia bioestratigráfica, paleobiogeográfica y paleoambiental. Universidad Nacional de La Plata, Tesis Doctoral, 367 p.

Zurita, A.E.; Miño-Boilini, A.R.; Soibelzon, E.; Carlini, A.A. \& Paredes-Ríos, F. 2009. The diversity of Glyptodontidae (Xenarthra, Cingulata) in the Tarija Valley (Bolivia): systematic, biostratigraphic and paleobiogeographic aspects of a particular assemblage. Neues Jahrbuch für Geologie und Paläontologie, Abhandlungen, 251:225-237. doi: 10.1127/0077-7749/2009/0251-0225

Received in June, 2011; accepted in March, 2012. 